\title{
A developed version of the Hooke's law
}

\author{
Zdzislaw Pluta, Tadeusz Hryniewicz \\ Koszalin University of Technology, Raclawicka 15-17, PL 75-620 Koszalin, Poland \\ E-mail address: Tadeusz.Hryniewicz@tu.koszalin.pl
}

\author{
"... ut tensio sic vis ..." \\ (...such an elongation as the force is... \\ here: the force increment - by Z.P.) \\ Robert Hooke
}

\begin{abstract}
The paper contains a proper approach to the Hooke's law as well as its developed version. A system approach was applied covering all the process of material elongation. An adequate interpretation of this law is given indicating that it touches only changes of magnitudes which describe the considered reality. The plot of elongation curve has been presented by separating a series of proper zones connected with the phenomena occurring in this process. A developed version of the Hooke's law, taking into account the considered reality concerning the material stress both its own as well as caused by external loading, is the conclusion of these considerations.
\end{abstract}

Keywords: Hooke's law; System approach; Deformation zones; Zones of forces and stresses; Rigidity; Coefficient of elastic rigidity; Young's modulus; Modulus of own/particular elasticity

\section{SYSTEM APPROACH TO THE PROBLEM}

A system approach functions nowadays as "the skill/competence of total perception and description of the reality" [1]. In the development of this definition [2] one should take into account particular components of the system and interactions occurring between them. System approach requires, however, turning into the properties of the system, its structure and function, and its evolution [1].

The system approach has been introduced in different zones of human activity. This approach is also considered in the international standarization systems [3]. In [4] there is a system approach assumed as the study method referred to the qualitative evaluation of the grinding wheels used to finishing operations of the ring rolling bearings on the grinding automatic machine.

The work [3] presents a part of exemplary system approach in explaining improvement of an automobile braking system. Apart from the properties of brake shoes and brake disks, there are other elements and agents that should be taken into consideration even those being out of the system, like the weather, state of the roads, or the driver conditions. All they interact and may affect the considered system. 
The authors of this work would like to introduce this system thinking to the scientific problems concerning the existent theory. Let us reflect the discussed subject to a proper understanding of the Hooke's law and its developed version.

\section{STRETCHING CURVE AND THE HOOKE'S LAW}

The elongation curve is closely related with the Hooke's law, as it describes just the initial fragment presenting the phenomenon of elastic deformation of a material under extension. The system approach to the considered problem requires presentation of the extension process as just after this the inefficiency of existing description can be revealed evidently. At first the evaluation of the existent description of extension curve and its initial linear part is considered. Then the shortcomings of this description and next the adequate approach to the problem will be discussed.

A typical curve of extension, characterizing the process of extension of ductile materials has quite a complex configuration (Fig. 1). This curve used to be presented in the coordinates $F-\Delta l$ (force - deformation), or in coordinates $\sigma-\varepsilon$ (stress - relative deformation). These first coordinates are essential for a given sample/element, the second one - to determine resistance properties of a material.

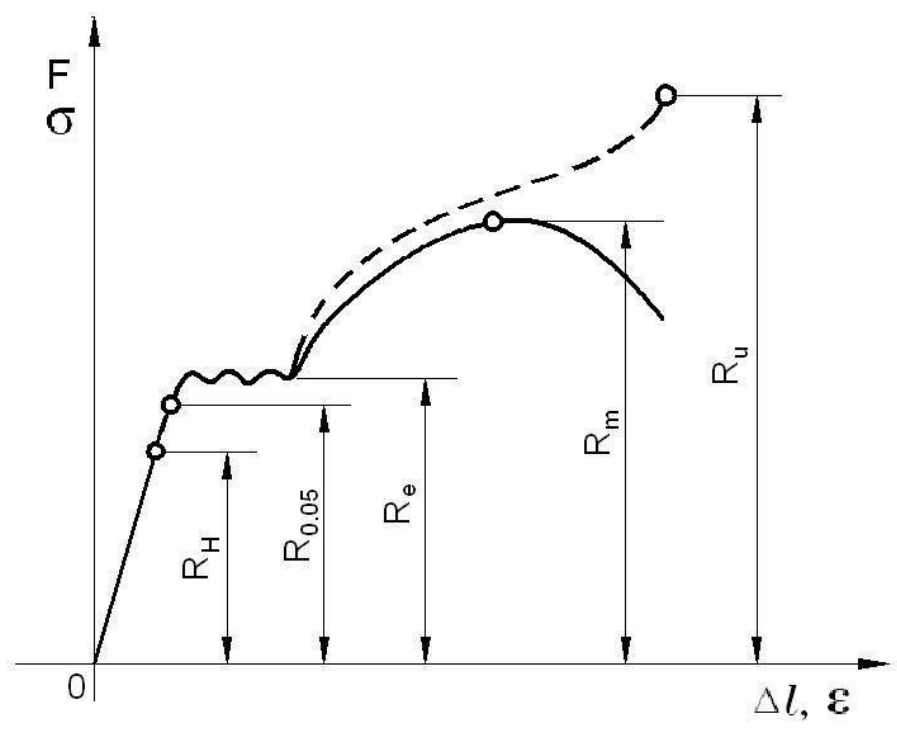

Fig. 1. Typical tensile curve

At the beginning the course of the plot is linear with loading, proportional to the deformation. An analogous connection is formed by stress and deformation and the corresponding phenomenon observed and discovered first time in the $17^{\text {th }}$ century by $\mathrm{R}$. Hooke is known as the law under his name. It forms the elastic theory and appears in the range of stresses higher than $R_{H}$, known as the limit of proportionality. Deformation of a material in this range is characteristic with its reversibility, i.e. the sample dimensions return to their initial state after the loading removal.

Under a further deformation the plastic strain/permanent set begins to occur which does not disappear/vanish after the loading removal. The stress corresponding with the plastic strain is equal $0.05 \%$ of the initial length of sample and is called the elastic limit and denoted by symbol $R_{0.05}$. After crossing the elastic limit the higher and higher plastic strains occur and the stress reaches the yield point $R_{e}$ with a rapid increase of deformation: the material flow 
appears afterwards. Further deformation is accompanied with - first, increase, and after crossing some maximum value $R_{m}$ (tensile strength) of the stipulated/agreed upon stresses their decrease; in one place there is the sample necking/narrowing ended with its fissure/rupture.

Decrease of the stipulated/agreed upon stresses results from their reference to the initial sample cross-section $A_{o}$. All other up-to-date magnitudes marked on the solid line are referred to this cross-section. The dashed line on this plot presents the character of changes of real stresses from the moment of sample necking appearance. The last stresses are referred to the real cross-section $A$, and final, disrupted one $\left(R_{u}\right)$ - referred to the area of the sample's least cross-section, that is the ruptured sample.

After crossing the yield point (see dashed line in Fig. 1) the phenomenon of material strengthening/consolidation occurs. This is referred to chocking/keying of atoms in their new positions and mutual blocking of crystal grains (difference in sliding planes inclination and grain sizes and contamination). At the beginning this phenomenon is mild, a degressive course of the curve is observed, then it is rapid in its character, reflected by a progressive course of the further part of dashed line. This is the stage of stretching where the sample is disrupted evidently.

Let us present the higher given notation: $F$ - tensile force; $\Delta l$ - sample elongation; $\sigma_{r}-$ elongation stresses $\left(\sigma_{r}=F: A\right) ; \varepsilon$ - relative elongation $\left(\varepsilon=\Delta l: l_{o}\right)$, being the ratio of elongation $\Delta l$ to the initial length $l_{o}$ of sample.

The Hooke's law was formulated on the right proportional dependence between the tensile force $F$ and elongation $\Delta l$ of sample, with the extending of this dependence also onto a fictional zone (Fig. 2). The reality has been straightened and elongated into the direction of fictional straight-linearity.

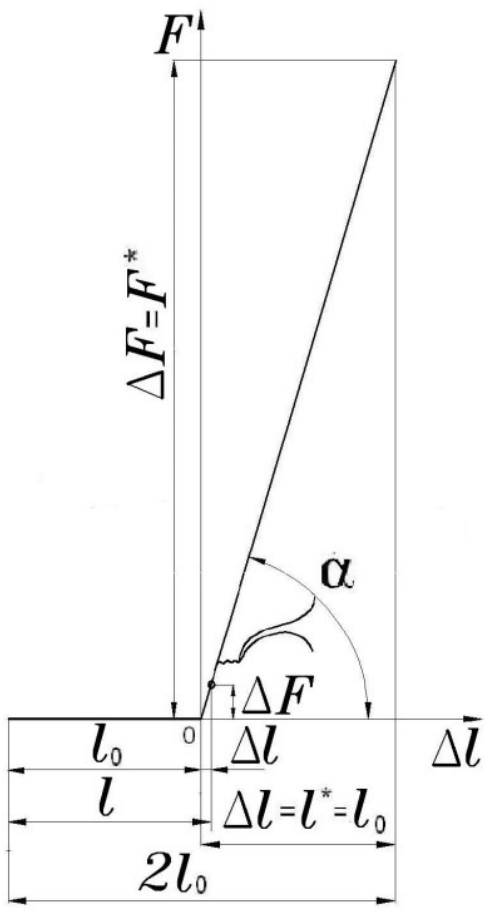

Fig. 2. Graphical interpretation of existent Hooke's law

This functional course is characteristic with the elastic/spring rigidity coefficient $k_{s}$, being in fact the tangent of angle $\alpha$, the angle of straight line inclination against axis $\Delta l$. This coefficient corresponds to the ratio of force $F$ (more exactly, the force increment $\Delta F$ ) and the 
length increment $\Delta l$ of the stretched sample. The tangent of the same angle $\alpha$ is the ratio of fictional force increment $\left(\Delta F=F^{*}\right)$ to the fictional length increment $\left(\Delta l=l^{*}=l_{o}\right)$, then equal to the initial length $l_{o}$.

One may write as follows:

$$
k_{s}=\frac{\Delta F}{\Delta l}=\frac{F^{*}}{l_{o}}
$$

From this proportion one may determine $\Delta l$, then:

$$
\Delta l=\frac{\Delta F \cdot l_{o}}{F^{*}}
$$

Let us divide and multiply the denominator by the area of initial cross-section $A_{o}$, and obtaining:

$$
\Delta l=\frac{\Delta F \cdot l_{o}}{\frac{F^{*}}{A_{o}} \cdot A_{o}} .
$$

Relation $F^{*}: A_{o}$ is the increment of fictional tensile stress which has been named by the Young's modulus denoted by symbol $E$. Then:

$$
\Delta l=\frac{\Delta F \cdot l_{o}}{E \cdot A_{o}}
$$

The quotient $\Delta F: A_{o}$ is the increment of a real tensile stress $\Delta \sigma_{r}$. Finally, after dividing of the both sides of equation (4) by $l_{o}$, one obtains:

$$
\varepsilon=\frac{\Delta l}{l_{o}}=\frac{\Delta \sigma_{r}}{E}
$$

and further on:

$$
\Delta \sigma_{r}=E \cdot \varepsilon
$$

This way the results of creative activities of Hooke's and Young's may be summed up. The results are, however, non-advantageous. The stretching phenomenon had been considered only until the moment of sample deformation appearance. De facto, only the co-dependence of increments: force $\Delta F$ and length $\Delta l$, tensile stress $\Delta \sigma_{r}$ and relative elongation $\varepsilon$, have been considered.

At this stage the authors of the work have introduced a correction to the Hooke's law. It refers to the increments of force and stress. In the next step of consideration this question will be developed and corrected from the point of view of agreement with the phenomenon nature.

The grounded classical description of this considered problem is generally known in the textbooks on materials endurance/strength. There are just a few of the vast literature cited in this point [5-7]. 


\section{TENSILE CURVE IN A PROPER COORDINATION SYSTEM}

The phenomenon of sample stretching begins from the loading moment. It is known that the element material possesses its internal stresses, occurring without external loading and they are in equilibrium inside some area. Therefore the sample will not undergo stretching from the very beginning. It will occur in time when the external loading fully surmounts that material resistance, resulting from its own internal stresses. Thus the tensile curve should have its beginning in a proper coordination system, i.e. connected with the beginning of stretching process with the following magnitudes as the coordinates: material resistance force $F$, sample elongation $\Delta l$, tensile stress $\sigma_{r}$, and relative elongation $\varepsilon$ of the sample.

Indicative course of these magnitudes (Fig. 3) is shifted against the up-to-date assumed position of the system of determined coordinates. Now considered are not the increments of forces and stresses but full forces of material resistance and its stresses. All these increments are increased of the own/internal resistance force $F_{o}$ and internal stresses $\sigma_{f o}$, that is connected with shifting of the system on these magnitudes. At this stage the beginning of system corresponds with the beginning of sample loading.

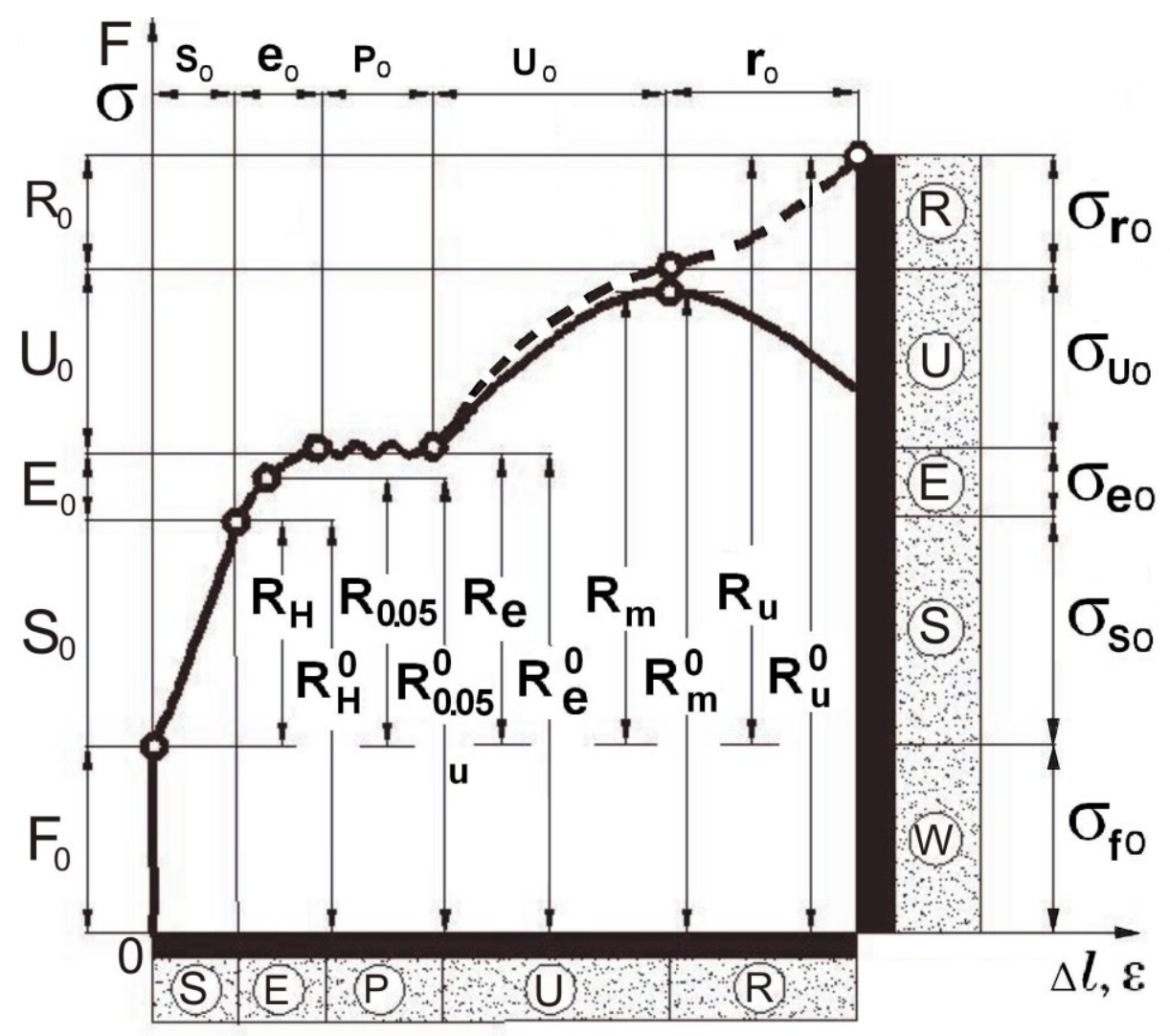

Fig. 3. The whole picture of tensile curve 
Therefore a necessity exists to properly correct the parameters of characteristics of stretching process. Instead of the up-to-date conventional/apparent parameters $\left(R_{H}, R_{0.05}, R_{e}\right.$, $R_{m}, R_{u}$ ), the following parameters are introduced: $R_{H}^{o}, R_{0.05}^{o}, R_{e}^{o}, R_{m}^{o}, R_{u}^{o}$. They are also resistances but increased of the own/internal stresses of material denoted by a symbol $\sigma_{f o}$. The new parameters are the real stresses, so they may be named: $R_{H}^{\circ}$ - real proportionality limit, $R_{0.05}^{o}$ - real elasticity limit, $R_{e}^{\circ}$ - real plasticity limit, $R_{m}^{o}$ - real tensile strength, $R_{u}^{\circ}-$ real burst/disruption stresses, respectively.

One may notice the tensile indicatrix has a complex configuration, containing particular points divided it into determined zones/strips both as to the forces and stresses, and deformations of the stretched material. In each of these zones quite a particular characteristic phenomenon occurs. Its name decides of the zone name where this phenomenon takes place.

The following zones are placed on the force/stress direction: own/internal (W), elastic $(\mathrm{S})$, resilient $(\mathrm{E})$, enforcement $(\mathrm{U})$, and disruption $(\mathrm{R})$. In the detailed classification, these zones (Fig. 4) have been divided, first into own and enforced. The last one covers the following zones: elastic, resilient, and neuralgic. The neuralgic zone is formed by two zones: reinforcement, and disruption.

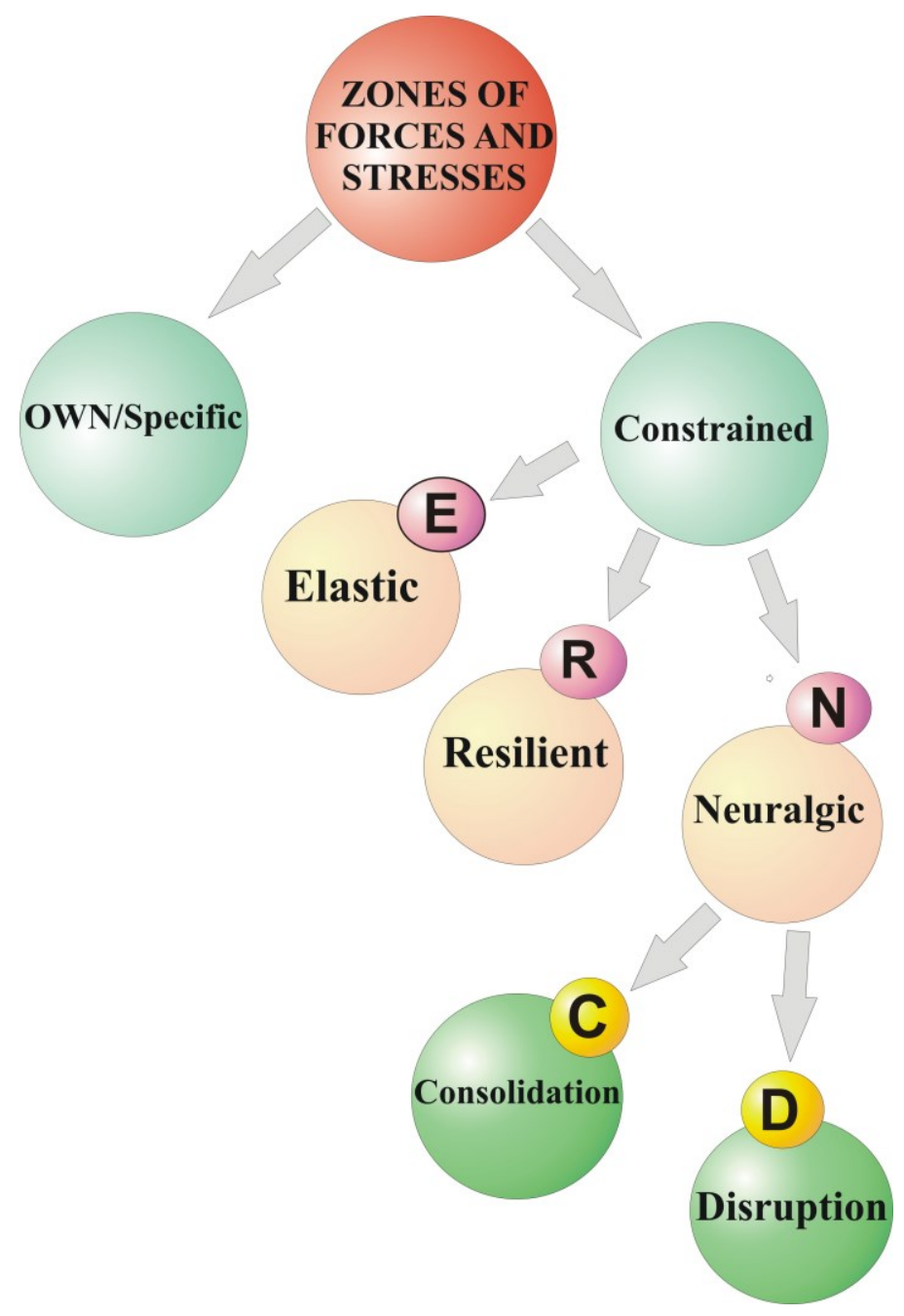

Fig. 4. The classification of zones of forces and stresses 
The direction of deformations is denoted by the following zones: elastic (S), resilient $(\mathrm{E})$, plastic $(\mathrm{P})$, reinforcement $(\mathrm{U})$, and rupture $(\mathrm{R})$. These zones have been specified in the classification of deformation zones (Fig. 5). Here the last two zones are named by neuralgic zones.

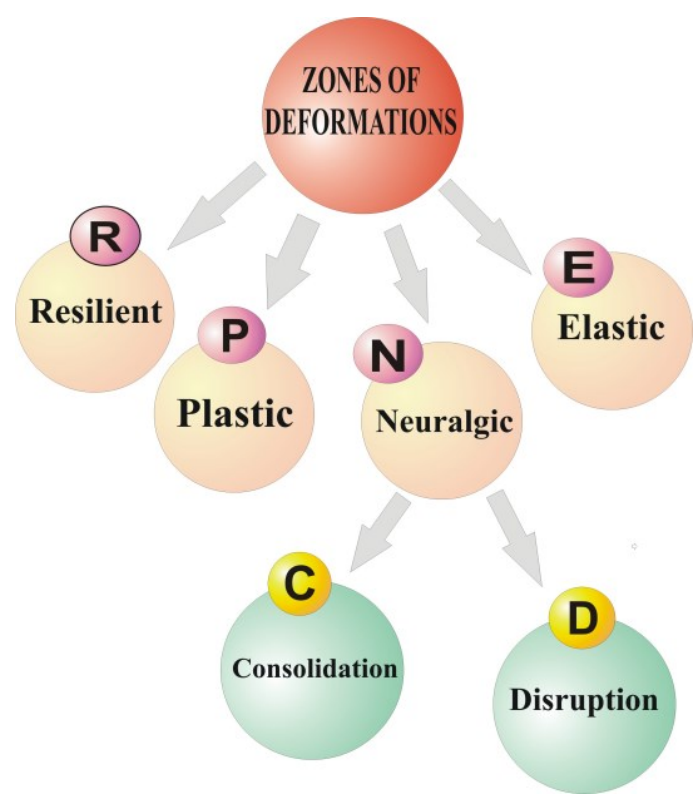

Fig. 5. The classification of deformation zones

A checkerwise pattern of zones spread over the directions of magnitudes characterizing the process of material stretching is presented in Fig. 5. The marked distinct limits of particular zones are passages between the neighbouring phenomena. On these passages some neighbouring material states co-exist timely meaning these sites have temporary/transient energetic character (quantum). It may be called the cascade system of limits and the performed observations indicate on the quantum nature of the considered part of reality.

Figure 5 presents also marking of sizes of particular zones. The overall dimensions magnitudes are forces (marked by capital letters with the index $o$ ), stresses (here denoted by sigma with double indicator: first referred to the sort of phenomenon, the second referred to the size of zone), and maximum components of length increments (symbol refers to the sort of phenomenon, and index $o$ is the same as mentioned above).

Such a denotation of zonal chain links of the material stretching process will ease the quantitative description of particular phenomena. This is not the subject of considerations but one description may be given as an example. This is the last neuralgic zone, i.e. the zone of stretching. Dependence of force $R$ on the elongation $r$ has the following form:

$$
R=R_{0}\left(e^{\frac{r}{r^{*}}}-1\right)
$$

where $r^{*}$ denotes the elongation constant. 
Similar character has the description of stresses, namely:

$$
\sigma_{r}=\sigma_{r o}\left(e^{\frac{r}{r^{*}}}-1\right)
$$

Such a detailed description of the tensile curve involves also next important open magnitude, the rigidity. Here are different rigidities (Fig. 6), dependent on zone and the phenomenon concerned. Measures of these rigidities are the determined coefficients. In reference to the elastic zone there is the coefficient of elastic rigidity $k_{s}$, described by formula (1), and in general form, by the differential $d S / d s$ (introduced herewith notations are connected with the description of the developed tensile curve).।

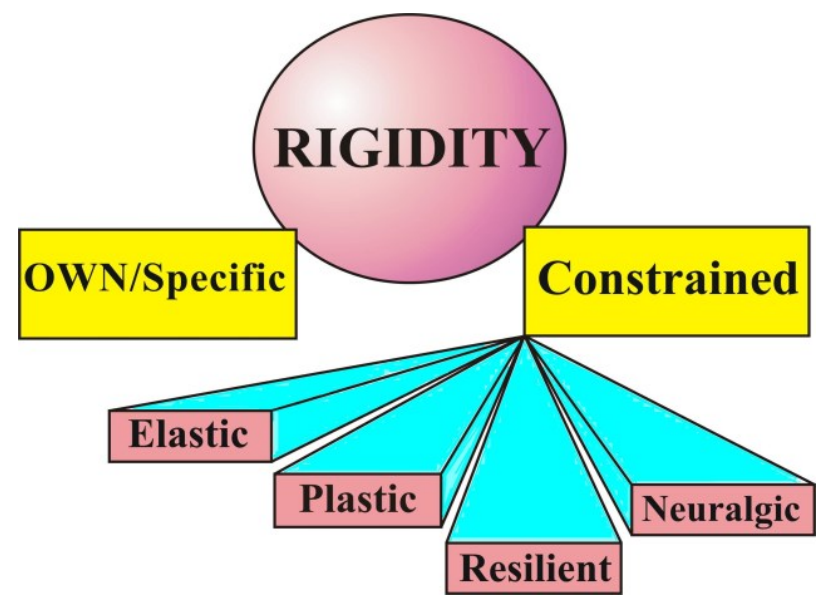

Fig. 6. Rigidity classification

\section{DEVELOPED VERSION OF THE HOOKE'S LAW}

The developed version of the Hooke's law results directly from the adequate complete description of the material stretching process. The complete stress $\sigma_{r}$ is the sum of the own/inside stress $\sigma_{f o}$ and the increment of stress $\Delta \sigma_{r}$, giving:

$$
\sigma_{r}=\sigma_{f o}+\Delta \sigma_{r}
$$

The own/inside stress $\sigma_{f o}$ may be determined on the basis of scheme (Fig. 7) and definition of the coefficient of elastic rigidity $k_{s}$, referred to the own/inside initial state of material and fictional state, determined by formula (1). Thus:

$$
k_{s}=\frac{F_{o}}{\Delta l_{o}}=\frac{F^{*}}{l_{o}}
$$




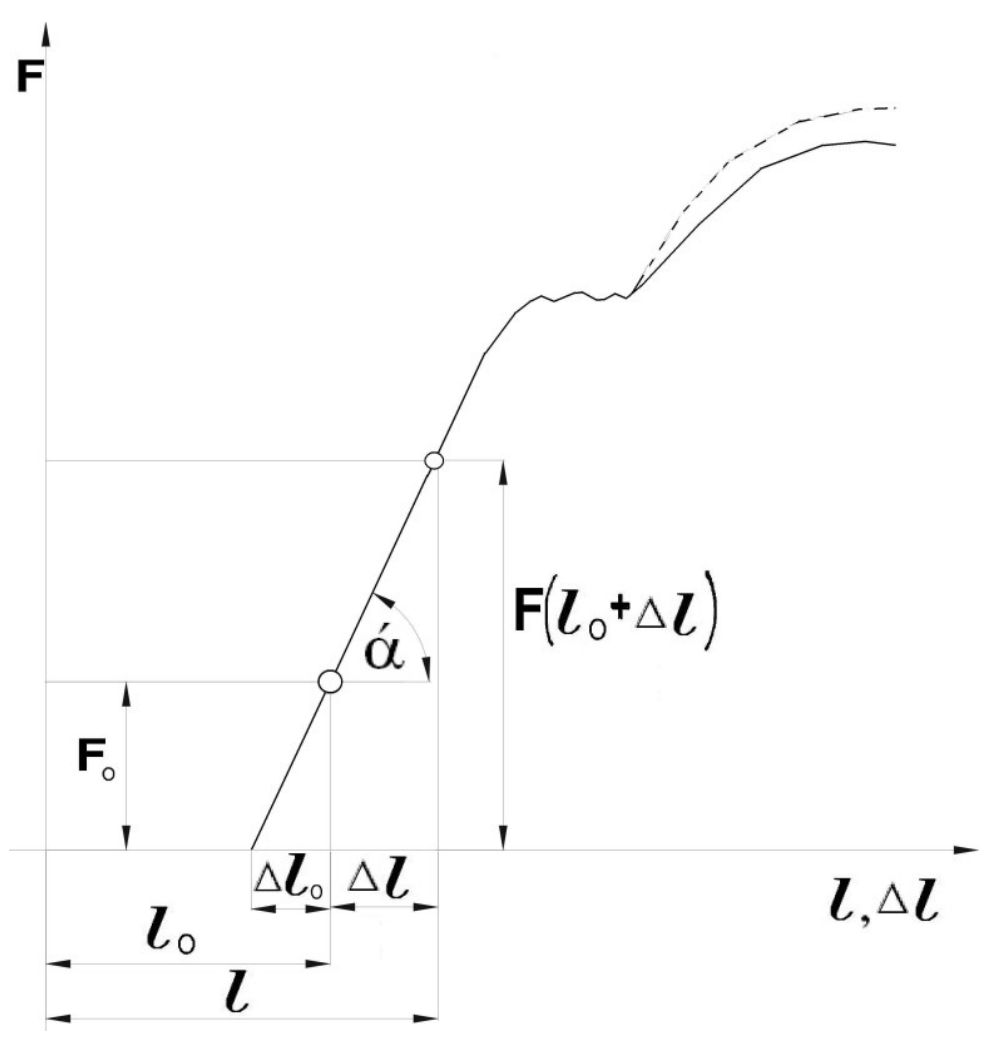

Fig. 7. Real course of tensile curve

From formula (10) the apparent material deformation $\Delta l_{o}$ is determined equal as to its value to that one which may appear at the increase of force of value equal to the initial force $F_{o}$. This magnitude is described by:

$$
\Delta l_{o}=\frac{F_{o} \cdot l_{o}}{F^{*}}
$$

By dividing and multiplication of the denominator through the initial area of sample cross-section, that is $A_{o}$, one obtains:

$$
\Delta l_{o}=\frac{F_{o} \cdot l_{o}}{\frac{F^{*} \cdot A_{o}}{A_{o}}}=\frac{F_{o} \cdot l_{o}}{E \cdot A_{o}}
$$

and then after dividing both sides of the equation by $l_{o}$ and taking into account, the quotient $F_{o}: A_{o}$ is the own/inside stress $\sigma_{f o}$ :

$$
\varepsilon_{o}=\frac{\sigma_{f_{o}}}{E}=\frac{E_{o}}{E}
$$

where $\varepsilon_{o}$ is the apparent relative deformation, and $E_{o}$ - modulus of own/internal elasticity (initial). 
From equation (13) it results that

$$
\sigma_{f o}=E_{o}=E \cdot \varepsilon_{o}
$$

By substituting (14) to (9), and taking into account (6) on the increment of stresses one obtains finally:

$$
\sigma_{r}=E_{o}+E \cdot \varepsilon
$$

and next, after taking into account the product (14), one obtains:

$$
\sigma_{r}=E\left(\varepsilon_{o}+\varepsilon\right)
$$

This is the developed version of the Hooke's law. It takes into consideration the whole considered reality, i.e. the material stresses both its own/internal and those caused by an external loading.

\section{CONCLUSION}

Each considered problem, not only presented in this work, if treated by a system approach makes it possible to discover and describe another part of reality. The presented herewith the developed version of the Hooke's law is the result of such system in-depth complete considerations. It is worth following this trace to reveal the true description of reality.

To sum up it is worth underlining a very important question, being the essence of the scientific cognition. This is the positioning of coordination system. The adequate description of phenomenon in this system is possible when it is connected with the beginning of phenomenon. Otherwise only changes of a magnitude characterizing it may be described. This has a much wider meaning and is important not only during creation of strength characteristics of a material.

\section{References}

[1] G. J. Klir, General theory of systems. Development of tendencies (in Polish). WNT, Warszawa 1976.

[2] G. Ossimitz, The Development of systems thinking skills using system dynamics modeling tools, 1997; http://wwwu.uni-klu.ac.at/gossimit/sdyn/gdm_eng.htm

[3] E. Ślażyńska, System approach in standarization of works IEC (in Polish). Normalizacja, 11 (2007) 8-10.

[4] B. Słowiński B., Quality of abrasive tools in a system approach (in Polish). Materiały XXV Naukowej Szkoły Obróbki Ściernej. Łódź, September 2003, 73-78. 
[5] R. Kurowski, M. E. Niezgodziński, Materials strength (in Polish). 8th edition, PWN, Warszawa 1968.

[6] Z. Brzoska, Materials strength (in Polish). PWN, Warszawa, 1972.

[7] A. Jakubowicz, Z. Orłoś, Materials strength (in Polish). 5th edition, Warszawa, 1978. 